Andrew J. Parnell

\title{
Nanotechnology and the potential for a renewable solar future
}

ABSTRACT. Energy is a basic requirement for people in the modern world. Sadly, it is only going to become more expensive in the future as the world population increases and oil becomes harder to find. A renewable energy future is essential to mitigate both the rising cost of oil and the damage wrought by carbon dioxide to the Earth and its climate. Solar energy is a highly promising technology capable of supplying a large amount of our total energy needs with very few downsides. Solar cells use nanoscale architecture either as the morphology for the active component or by using nanoscale thin film architecture (or both). At present the majority of commercial solar cells are based on silicon technology. They are rigid panels that use complex and expensive processing methods to manufacture. Adopting new manufacturing methods based on nanoscale self-assembly could launch solar cells into one of the dominant energy-producing technologies over the next few years. In this article I discuss the possibility of mass-produced solar cells based on organic polymer solar cell materials and their potential to made at a fraction of the existing manufacturing costs and in much higher volume.

Nanotechnology Perceptions 7 (2011) 180-187

Nonsubscribers: purchase individual article 